Check for updates

Cite this: RSC Adv., 2018, 8, 22652

\title{
Extraction of isoflavones from Puerariae lobata using subcritical water
}

\author{
Hongguang Zhang, (DD ab Shuhua Liu, ${ }^{a}$ Huaizhi Li, ${ }^{\mathrm{C}}$ Fumin Xue, ${ }^{a}$ Shuxin Han, ${ }^{a}$ \\ Liang Wang, ${ }^{b}$ Yan Cheng (iD *a and Xiao Wang ${ }^{\star a}$
}

As an alternative to organic solvents, subcritical water was employed for the first time as an effective solvent for the extraction of isoflavones from Puerariae lobata. Optimum experimental conditions for the extraction of the four main isoflavones were established by single factor experiments, and the optimum experimental conditions for total isoflavone extraction were established further by response surface methodology. With an extraction time of $45 \mathrm{~min}$ and a liquid/solid ratio of $1: 20$, the extraction yields of puerarin, $3^{\prime}$ methoxypuerarin, and daidzin reached maxima at extraction temperatures of $120^{\circ} \mathrm{C}, 140{ }^{\circ} \mathrm{C}$ and $200{ }^{\circ} \mathrm{C}$, respectively. Moreover, puerarin, $3^{\prime}$-methoxypuerarin and daidzin were degraded and produced various byproducts due to hydrothermal reactions at higher temperatures. The maximum extraction yields of the total isoflavones were obtained by response surface methodology (extraction time of $45 \mathrm{~min}$, solid/liquid ratio of $1: 15$ and extraction temperature of $120{ }^{\circ} \mathrm{C}$ ). Compared to conventional solvents, subcritical water utilized less solvent and required a shorter extraction time.

Received 27th March 2018 Accepted 6th June 2018

DOI: 10.1039/c8ra02653j

rsc.li/rsc-advances extract isoflavones from Puerariae lobata on a large scale.,414-16 These extraction solvents are not environmentally friendly. Moreover, the extraction processes may easily leave solvent residues in the extracts. Water is a safer, cheaper and more easily available solvent than organic solvents. Furthermore, the physicochemical properties of water can be easily manipulated by changing the temperature or pressure. ${ }^{17}$ When water is heated under sufficient pressure to maintain it in a liquid state (i.e., pressurized hot water), there are significant changes to its polarity. Typically, the dielectric constant $(\varepsilon)$ of water at $25^{\circ} \mathrm{C}$ is approximately 80 , but it reduces to approximately 27 at $250^{\circ} \mathrm{C}$, which is similar to that of methanol $(\varepsilon=33$ at $298 \mathrm{~K})$ or ethanol $(\varepsilon=25$ at $298 \mathrm{~K}) .{ }^{18}$ The lower surface tension and viscosity of pressurized hot water also increase mass transfer rates of active compounds from the plant tissue matrix. These physicochemical properties of water are the basis for using subcritical water to replace organic solvents in extraction processes. Thus, subcritical water extraction (SCWE) has received significant attention from researchers over the past three decades as a feasible green alternative to organic solvents. ${ }^{19,20}$ Many extraction studies from herbs or food byproducts using SCWE have been reported. Ibaňez et al. investigated the effect of temperatures ranging from 25 to $200{ }^{\circ} \mathrm{C}$ on the selective extraction of antioxidant compounds from rosemary leaves. ${ }^{21}$ Subcritical water could extract most of the active compounds from rosemary, such as carnosol, rosmanol, carnosic acid, and methyl carnosate. Some extracts such as cirsimaritin and genkwanin showed high selectivity. Myong-Soo et al. studied SCWE of the flavonol quercetin from onion skin using a temperature range of $100-190{ }^{\circ} \mathrm{C}$ and extraction times of 5-30 min. ${ }^{14} \mathrm{Using}$
${ }^{a}$ Key Laboratory of TCM Quality Control Technology, Shandong Analysis and Test Center, Qilu University of Technology (Shandong Academy of Sciences), Jinan 250014, China.E-mail:wangx@sdas.org; chengyan99@163.com

${ }^{b}$ College of Chemistry and Chemical Engineering, Ocean University of China, Tsingtao, Shandong, 266100, China

'The People's Hospital of Junan, Linyi, Shandong, 276600, China 
SCWE, the quercetin yield was over eight-, six-, and four-fold greater than those obtained using the ethanol, methanol and water-at-boiling-point extraction methods, respectively. Depending on the SCWE conditions, it was possible to obtain targets with different chemical compositions.

Response surface methodology (RSM) is an effective statistical technique for optimizing multiple, inter-related parameters. ${ }^{22-24}$ It is less laborious and time-consuming than other methods that have been applied to process optimization. Therefore, it has been widely used to optimize the extraction processes of active compounds from herbs or food byproducts. $^{25,26}$

This study investigated subcritical water as a feasible processing solvent for extraction of four isoflavone components and total isoflavones from Puerariae lobata. SCWE parameters, such as extraction temperature, extraction time and solid/liquid ratio, were systematically optimized by RSM for enhancing the extraction efficiency. The objective of the present work was to achieve rapid, and environmentally friendly water extraction conditions for four isoflavone components and the total isoflavonoids from Puerariae lobata.

\section{Materials and methods}

\subsection{Chemicals and reagents}

Reference standards of puerarin, $3^{\prime}$-methoxypuerarin, daidzin and daidzein (purity $\geq 98 \%$ ) were supplied by Shanghai Yuanye Biotechnology Co., Ltd, China. Methanol and formic acid (Analytical Grade, Tianjin, China) were purchased from Tianjin Kermel Chemical Reagent Co., Ltd. Acetonitrile (Tedia Company Inc., Fairfield, USA) was used for HPLC analysis. Deionized water $(>18 \mathrm{M} \Omega \mathrm{cm})$ was prepared using an Elga Purelab water system (Elga, England).

Dry Puerariae lobata was purchased from the Zhonglu Hospital. The roots of Puerariae lobata were washed, dried and ground in a grinder (Zhongxing Weiye, Beijing, China). The powder was passed through a 60 mesh sieve and stored at $-4{ }^{\circ} \mathrm{C}$.

\subsection{Experimental design and statistical analysis}

The experiments were executed in two phases. The objective of phase I was to obtain the optimum extraction conditions: extraction temperature, extraction time and ratio of solid/liquid for four isoflavone components in Puerariae lobata. The main role of the pressure was to maintain the water in liquid form at high temperature. Therefore, the appropriate pressure was maintained for each given temperature. In our study, water was mainly present in liquid form in our investigated temperature range. In this phase, subcritical water with a temperature range of $100-200{ }^{\circ} \mathrm{C}$ was investigated at $20^{\circ} \mathrm{C}$ intervals. The extraction temperature of $120{ }^{\circ} \mathrm{C}$ was selected for a range of extraction times from $15 \mathrm{~min}$ to $75 \mathrm{~min}$. The reason for the selection of $120{ }^{\circ} \mathrm{C}$ is described in the Results and discussion. Extraction yields of puerarin, $3^{\prime}$-methoxypuerarin, daidzin and daidzein were determined from the corresponding standard curves obtained by HPLC analysis. Stock solutions of puerarin, 3'- methoxypuerarin, daidzin and daidzein standards were prepared in methanol at concentrations of $60 \mu \mathrm{g} \mathrm{mL} \mathrm{m}^{-1}$. Working standard solutions were prepared in the range of 3-15 $\mu \mathrm{g} \mathrm{mL} \mathrm{m}^{-1}$ by diluting the appropriate stock solution with methanol, and were stored at $-4{ }^{\circ} \mathrm{C}$ in darkness. The objective of phase II was to obtain the optimum extraction conditions (extraction temperature, extraction time and ratio of solid/ liquid) for the total isoflavones in Puerariae lobata by using RSM. On the basis of the results of phase I, the phase II experiment for total isoflavones was conducted over a narrower range of extraction temperatures $\left(110{ }^{\circ} \mathrm{C}, 120^{\circ} \mathrm{C}\right.$ and $\left.140{ }^{\circ} \mathrm{C}\right)$ and extraction times $(15,30$ and $45 \mathrm{~min})$ at three different solid/ liquid ratios $(1: 10,1: 15$ and $1: 25)$. To further study the interactions between the extraction parameters, RSM was employed to obtain the optimum operating parameters. The Box-Behnken program was used to design experimental projects, analyze statistical data and calculate the regression model. The statistical significance was checked using an $F$-test. The experimental extraction yields of total isoflavones were compared with those predicted from the RSM.

\subsection{Extraction procedures}

2.3.1 Subcritical water extraction. A $10 \mathrm{~mL}$ cylindrical stainless steel high-pressure batch extractor with an XTD-7000 isothermal furnace (Haian, China) was used for extraction of isoflavones from Puerariae lobata. A $0.20 \mathrm{~g}$ sample of dry Puerariae lobata powder was extracted with subcritical water in an extractor for the designated time at the designated temperature. Then the extractor was taken out of the furnace, cooled in water and opened. Extracts were collected with methanol. We paid much attention to the selection of the methanol solvent to dissolve the four isoflavones. On the one hand, the dielectric constant of water at $200^{\circ} \mathrm{C}$ is similar to that of methanol. When the extracts were cooled to room temperature, we hypothesized that the utilization of methanol with its low dielectric constant might prevent the target extracts from insolubility. On the other hand, some researchers have utilized methanol to extract the isoflavone compounds ${ }^{27,28}$ and we could refer to their experimental procedures. Methanol was added to make the required final volume and the solution was filtered through a $0.45 \mu \mathrm{m}$ Nylon syringe filter (Jinteng, Tianjin) for HPLC analysis.

2.3.2 Reflux extraction. $\mathrm{Zhu}^{28}$ has previously investigated the influence of extraction temperature, time and ratios of ethanol-water, and described a reflux extraction procedure at an extraction temperature of $90{ }^{\circ} \mathrm{C}$, extraction time of $120 \mathrm{~min}$ and extraction solvent of $70 \%$ ethanol-water. We referred to these published experimental conditions in developing a typical reflux extraction procedure as follows: $1.00 \mathrm{~g}$ aliquot of dry Puerariae lobata powder was extracted with $30 \mathrm{~mL} 70 \%$ ethanolwater solvent at $90{ }^{\circ} \mathrm{C}$ for $120 \mathrm{~min}$ in a round-bottom flask with a Dimroth condenser in a thermostatic waterbath. Extracts were transferred into a $100 \mathrm{~mL}$ volumetric flask and methanol was added to make the final volume. This extraction procedure was repeated three times.

2.3.3 Ultrasonic extraction. $\mathrm{Li}^{29}$ has investigated the influence of temperature and time for ultrasonic extraction and the 
optimal extraction procedure was confirmed to be $50{ }^{\circ} \mathrm{C}$ and 45 min. Thus we used a typical extraction procedure with ultrasonic extraction as follows: $0.20 \mathrm{~g}$ dry Puerariae lobata powder was extracted with distilled water in an ultrasonic waterbath at $50{ }^{\circ} \mathrm{C}$ for $45 \mathrm{~min}$ (KQ-600 KDE, Kunshan, China). The extracts were combined and then centrifuged at $10000 \mathrm{rpm}$ for $10 \mathrm{~min}$. This extraction procedure was repeated three times.

\subsection{HPLC apparatus and operating conditions}

An UltiMate 3000 liquid chromatography system (Thermo Fisher Scientific Inc., Waltham, USA) with a five binary gradient pump, UV detector, column oven and autosampler was used for detection of puerarin, $3^{\prime}$-methoxypuerarin, daidzin and daidzein. Each sample solution $(10 \mu \mathrm{L})$ was injected into a C 18 column ( $25 \mathrm{~cm} \times 4.6 \mathrm{~mm}, 5 \mu \mathrm{m}$, InertSustain). Detection was at $260 \mathrm{~nm}$ and $35{ }^{\circ} \mathrm{C}$. The mobile phase consisted of two components: (A) $0.1 \%$ formic acid in water, and (B) acetonitrile. The gradient elution program was as follows: $15-30 \% \mathrm{~B}$ (0-16 min), $30-100 \%$ B (16-34 min), 100-100\% B (34-40 min), 100-15\% B
(40-41 $\mathrm{min}), 15-15 \% \mathrm{~B}(41-42 \mathrm{~min})$. The flow rate was $0.80 \mathrm{~mL} \min ^{-1}$.

\subsection{Statistical analysis}

Calibration curves of puerarin, $3^{\prime}$-methoxypuerarin, daidzin and daidzein were used for calculating the extraction yields. The optimum SCWE conditions, i.e., extraction temperature, extraction time and ratio of solid/liquid from each experiment were chosen based on the highest isoflavone contents. Three replicates of each analysis were performed in order to determine the reproducibility of the procedure. Results are expressed as mean \pm SD. Statistical comparisons were made by one-way analysis of variance (ANOVA). Differences were considered to be significant when the $p$ values were $<0.05$.

\section{Results and discussion}

\subsection{HPLC chromatograms}

Fig. 1b displays HPLC chromatograms of standard substances including puerarin, $3^{\prime}$-methoxypuerarin, daidzin and daidzein
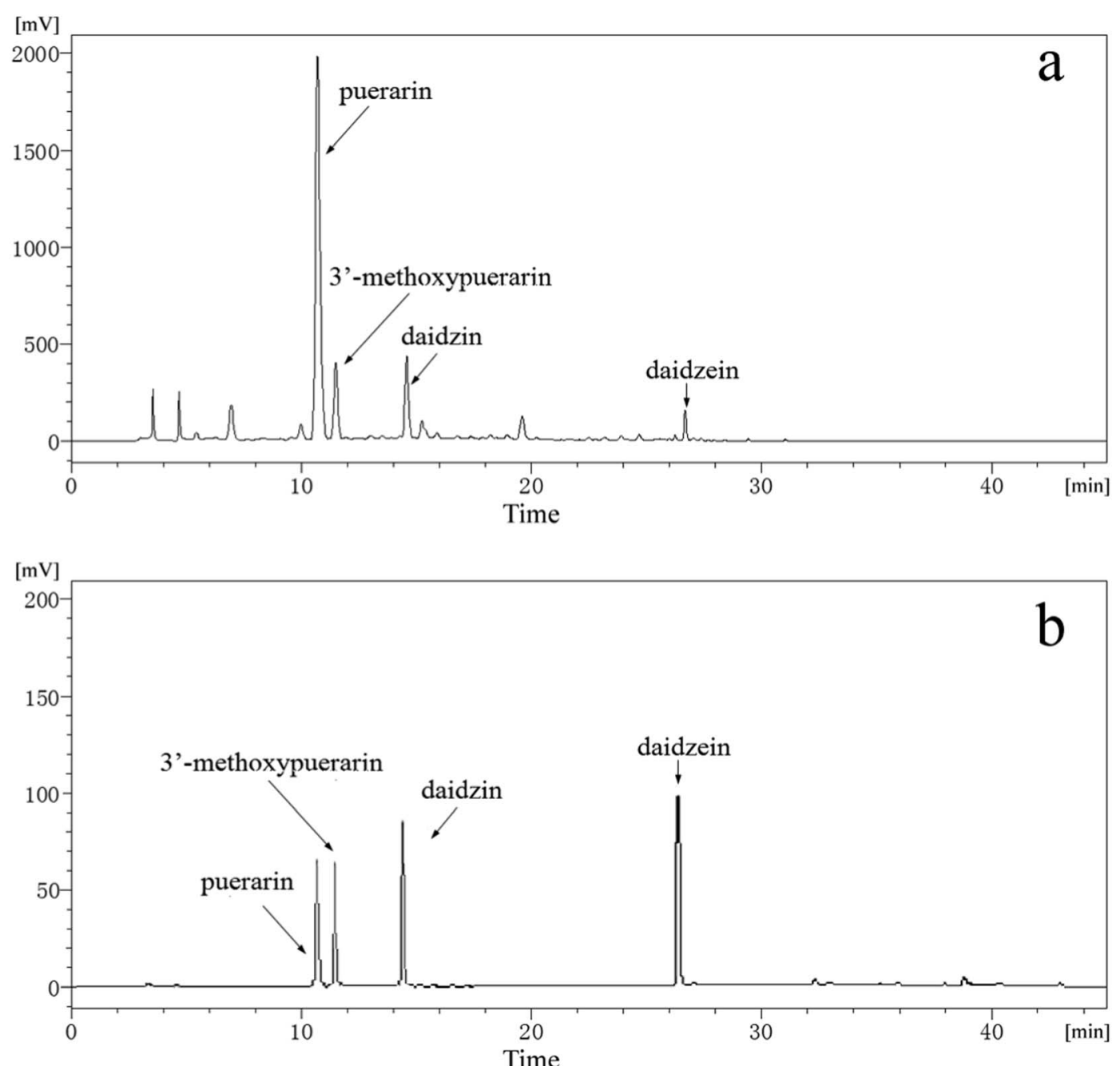

Fig. 1 (a) Typical HPLC chromatogram of the Puerariae lobata extract obtained by SCWE (120 ${ }^{\circ} \mathrm{C} ; 15 \mathrm{~min} ; 4.50 \mathrm{~mL}$ water); (b) HPLC chromatogram of standard substances (puerarin, $3^{\prime}$-methoxypuerarin, daidzin and daidzein) mixed at concentrations of $10 \mu \mathrm{g} \mathrm{mL}$. 
mixed at concentrations of $10 \mu \mathrm{g} \mathrm{mL}^{-1}$. As shown in Fig. 1b, the retention times of the four standard substances are 10.8, 11.8, 14.4 and $26.6 \mathrm{~min}$, respectively. Fig. 1a shows a typical chromatogram of the Puerariae lobata extract obtained using SCWE at $120{ }^{\circ} \mathrm{C}$ for $15 \mathrm{~min}$ with $4.5 \mathrm{~mL}$ water. By comparing the retention times and UV spectra with those of the reference standards, four main peaks corresponding to puerarin, 3'methoxypuerarin, daidzin and daidzein were identified. Other minor peaks were not identified in this study because of the lack of suitable standards. Thus, puerarin, 3'-methoxypuerarin, daidzin and daidzein were chosen as the objective compounds for the study.

\subsection{Calculation of total isoflavone yield}

The yields of puerarin, $3^{\prime}$-methoxypuerarin, daidzin and daidzein were obtained using the following five concentrations: 3.0, $6.0,9.0,12.0$, and $15.0 \mu \mathrm{g} \mathrm{mL}{ }^{-1}$. The standard curves obtained corresponded to $y=17644 c_{1}-2725.7\left(R^{2}=0.9998\right)$ for puerarin; $y=21514 c_{2}-4239.5\left(R^{2}=0.9996\right)$ for $3^{\prime}$-methoxypuerarin; $y=33830 c_{3}-21418\left(R^{2}=0.999\right)$ for daidzin; and $y=$ $33830 c_{4}-21418\left(R^{2}=0.9991\right)$ for daidzein (where $y$ is the peak area from HPLC chromatograms and $c$ is the concentration of the target extracts). The extraction yields of the extracts were calculated with the following equation:

$$
\omega=c_{X} \times \frac{v}{m} \times 100 \%
$$

where $\omega$ is the extraction yield; $c_{X}$ is the concentration of the target extract $\left(X=1,2,3,4\right.$, corresponding to puerarin, $3^{\prime}$ methoxypuerarin, daidzin and daidzein, respectively); $v$ is the final collection volume of the extract with methanol and $m$ is the mass of Puerariae lobata. Herein, the total isoflavone yield was the summation of the extraction yields of puerarin, $3^{\prime}$-methoxypuerarin, daidzin and daidzein. The contents of all the extracts were measured using HPLC, and a spiking test was used to ensure that an accurate qualitative analysis was performed.

\subsection{Effect of extraction temperature on the yields of the four isoflavones}

The extraction temperature is an important factor that could influence the mass transfer rate and solubility of the isoflavones. As shown in Fig. 2, the extraction temperature range of 100-200 ${ }^{\circ} \mathrm{C}$ was chosen. When the extraction temperature increased from 100 to $120{ }^{\circ} \mathrm{C}$, the extraction yields for puerarin and $3^{\prime}$-methoxypuerarin increased slightly. When the extraction temperature increased from 120 to $200{ }^{\circ} \mathrm{C}$, the extraction yields for puerarin and $3^{\prime}$-methoxypuerarin decreased obviously. Daidzin showed a similar trend but reached a maximum at $160{ }^{\circ} \mathrm{C}$. However, the extraction yield of daidzein remained constant from 100 to $160{ }^{\circ} \mathrm{C}$ and increased sharply when the extraction temperature exceeded $160{ }^{\circ} \mathrm{C}$. From the structures of puerarin and $3^{\prime}$-methoxypuerarin, we could deduce that there was an 8- $\beta$-D-glucopyranoside bond between the isoflavone skeleton and glucoside, so it was facile to conclude that the glucopyranoside bond was unstable and began to break down when the extraction temperature exceeded $120{ }^{\circ} \mathrm{C}$. As for

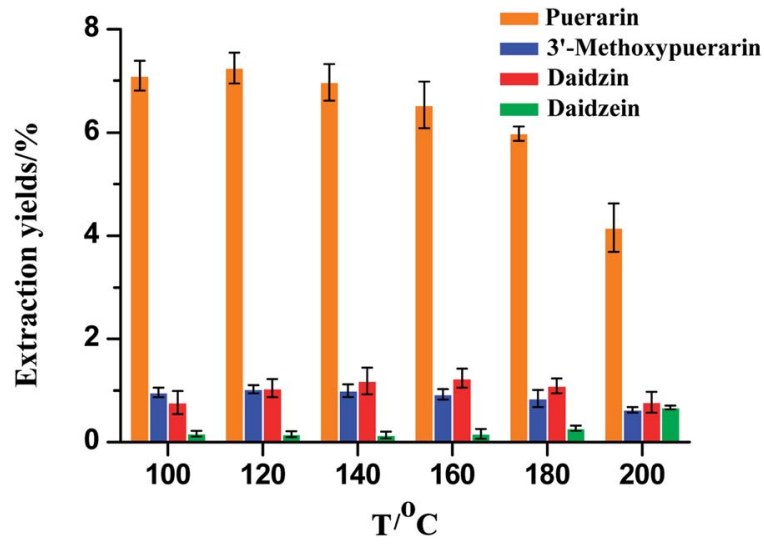

Fig. 2 Effect of extraction temperature on the extraction yields of isoflavones from Puerariae lobata (30 min; $4.50 \mathrm{~mL}$ water).

daidzin, there was an oxygen bond between the isoflavone skeleton and glucoside. When the extraction temperature exceeded $160{ }^{\circ} \mathrm{C}$, the oxygen bond became unstable and so the extraction yield of daidzin reached a maximum at $160{ }^{\circ} \mathrm{C}$. In contrast there was a relatively stable isoflavone skeleton for daidzein. At $160{ }^{\circ} \mathrm{C}$, daidzin degraded and produced daidzein, so the extraction yield of daidzein increased obviously.

\subsection{Effect of extraction time on the yields of the four isoflavones}

Extraction time is another important parameter for the extraction process. In the phase I experiment, Puerariae lobata powders were extracted at $120{ }^{\circ} \mathrm{C}$ for $15,30,45,60$ and $75 \mathrm{~min}$, as shown in Fig. 3. In general, the extraction yields of puerarin, $3^{\prime}$-methoxypuerarin and daidzin significantly increased when the extraction time increased from 15 to $45 \mathrm{~min}$. After $45 \mathrm{~min}$, an extension of the extraction time could not significantly improve the extraction yields of puerarin, $3^{\prime}$-methoxypuerarin and daidzin, however, the extraction yield of daidzein actually increased a little. These results indicated that the optimal extraction time for the four main isoflavones was between 30 and $45 \mathrm{~min}$.

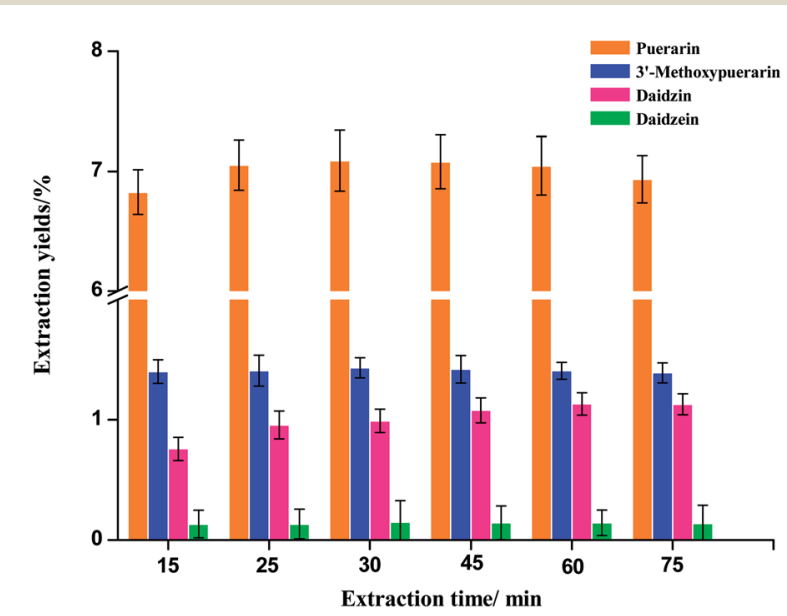

Fig. 3 Effect of extraction time on the extraction yields of isoflavones from Puerariae lobata $\left(120^{\circ} \mathrm{C} ; 4.50 \mathrm{~mL}\right.$ water). 


\subsection{Effect of solid/liquid ratio on the yields of the four isoflavones}

The effect of various water loadings on the extraction yields of the four isoflavones is shown in Fig. 4. In general, the use of a solid/liquid ratio from $1: 10$ to $1: 35$ at $120^{\circ} \mathrm{C}$ and $30 \mathrm{~min}$ had a small effect on the extraction yields of $3^{\prime}$-methoxypuerarin, daidzin and daidzein. The extraction yield of puerarin increased when the solid/liquid ratio increased from $1: 10$ to $1: 15$ and then decreased slightly when the solvent dosage increased over $1: 15$. The reason for the trend may be as follows: when the extract solvent volume increased to $4 \mathrm{~mL}$, the isoflavones could be completely extracted. However, a further increase of the water volume could decrease the solubility of puerarin, $3^{\prime}$ methoxypuerarin and daidzein. Thus, the optimal solid/liquid ratio of $1: 15\left(\mathrm{~g} \mathrm{~mL}^{-1}\right)$ was selected.

\subsection{Parameters optimized by RSM for total isoflavones}

To further study the interactions between the experimental factors, we optimized the extraction temperature, extraction time and solid/liquid ratio with RSM. The symbols and levels are shown in Table 1. The results are given in Table 2. The experimental values of extraction yield of total isoflavones from Puerariae lobata were analyzed by multiple regressions to fit the second order regression equation, and the regression model in terms of coded factors was predicted as follows (Table 4, eqn (2)):

$$
\begin{aligned}
Y= & 10.16+0.0075 x_{1}+0.096 x_{2}+0.026 x_{3} \\
& -0.021 x_{1} x_{2}+0.003632 x_{1} x_{3}-0.066 x_{2} x_{3} \\
& -0.18 x_{1}{ }^{2}-0.054 x_{2}{ }^{2}-0.11 x_{3}^{2}
\end{aligned}
$$

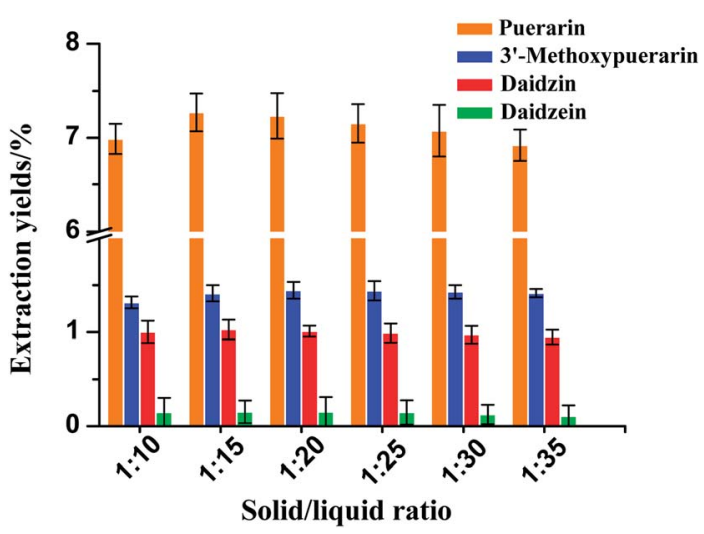

Fig. 4 Effect of solid/liquid ratio on the extraction yields of isoflavones from Puerariae lobata $\left(120^{\circ} \mathrm{C} ; 4.50 \mathrm{~mL}\right.$ water).

\begin{tabular}{|c|c|c|c|c|c|}
\hline \multirow[b]{2}{*}{ Factors } & \multirow[b]{2}{*}{ Units } & \multirow[b]{2}{*}{ Variables } & \multicolumn{3}{|c|}{ Range and levels } \\
\hline & & & -1 & 0 & 1 \\
\hline Extraction temperature & ${ }^{\circ} \mathrm{C}$ & $x_{1}$ & 110 & 120 & 140 \\
\hline Extraction time & $\min$ & $x_{2}$ & 15 & 30 & 45 \\
\hline Liquid/solid ratio & $\mathrm{g} \mathrm{mL}^{-1}$ & $x_{3}$ & $1: 10$ & $1: 15$ & $1: 25$ \\
\hline
\end{tabular}

Table 1 Experimental domain with natural and coded values of independent variables used in Box-Behnken design (BBD)
Table 2 Box-Behnken program experimental design with natural and

\begin{tabular}{|c|c|c|c|c|c|}
\hline \multirow[b]{2}{*}{ Run } & \multirow{2}{*}{$\begin{array}{l}\text { Temperature } \\
x_{1}\left({ }^{\circ} \mathrm{C}\right)\end{array}$} & \multirow{2}{*}{$\begin{array}{l}\text { Time } \\
x_{2} \\
(\min )\end{array}$} & \multirow{2}{*}{$\begin{array}{l}\text { Liquid/ } \\
\text { solid ratio } \\
x_{3} \\
\left(\mathrm{~g} \mathrm{~mL}^{-1}\right)\end{array}$} & \multicolumn{2}{|l|}{ Yield (\%) } \\
\hline & & & & $\begin{array}{l}\text { Actual } \\
\text { value }\end{array}$ & $\begin{array}{l}\text { Predicted } \\
\text { value }\end{array}$ \\
\hline 1 & 1 & 0 & 1 & $9.83 \pm 0.31$ & $9.84 \pm 0.30$ \\
\hline 2 & 0 & 0 & 0 & $10.02 \pm 0.22$ & $10.14 \pm 0.21$ \\
\hline 3 & 0 & 0 & 0 & $10.14 \pm 0.32$ & $10.14 \pm 0.33$ \\
\hline 4 & 1 & 0 & -1 & $9.84 \pm 0.47$ & $9.90 \pm 0.48$ \\
\hline 5 & -1 & -1 & 0 & $9.80 \pm 0.45$ & $9.81 \pm 0.49$ \\
\hline 6 & 0 & 0 & 0 & $10.16 \pm 0.39$ & $10.14 \pm 0.40$ \\
\hline 7 & 0 & 1 & 1 & $10.10 \pm 0.41$ & $10.12 \pm 0.40$ \\
\hline 8 & 0 & 0 & 0 & $10.18 \pm 0.37$ & $10.14 \pm 0.36$ \\
\hline 9 & 1 & 1 & 0 & $10.02 \pm 0.44$ & $10.00 \pm 0.43$ \\
\hline 10 & 0 & -1 & -1 & $9.98 \pm 0.24$ & $9.96 \pm 0.23$ \\
\hline 11 & 0 & 0 & 0 & $10.21 \pm 0.30$ & $10.14 \pm 0.29$ \\
\hline 12 & 0 & 1 & -1 & $10.09 \pm 0.47$ & $10.04 \pm 0.46$ \\
\hline 13 & -1 & 1 & 0 & $9.98 \pm 0.29$ & $10.04 \pm 0.29$ \\
\hline 14 & 0 & -1 & 1 & $9.73 \pm 0.18$ & $9.78 \pm 0.17$ \\
\hline 15 & 1 & -1 & 0 & $9.90 \pm 0.31$ & $9.85 \pm 0.30$ \\
\hline 16 & -1 & 0 & 1 & $9.92 \pm 0.43$ & $9.84 \pm 0.42$ \\
\hline 17 & -1 & 0 & -1 & $9.88 \pm 0.27$ & $9.89 \pm 0.26$ \\
\hline
\end{tabular}
coded SCWE conditions and experimentally obtained values for total yields of isoflavones from Puerariae lobata

where $Y$ is the response value (the extraction yields of total isoflavones from Puerariae lobata) and $x_{1}, x_{2}$ and $x_{3}$ are shown in Table 2 . The significance of each coefficient was determined by using the $F$-test and $p$-value illustrated in Table 3. For any of the terms in the models, a large $F$-value and a small $p$-value indicated a more significant effect on the respective response variables. The parity plot in Fig. 5 shows a comparison between the experimental yields of total isoflavones from Puerariae lobata and the values calculated by eqn (2). A perfect fit of the model to the data would result in all points lying on the diagonal in Fig. 5. The coefficient of determination of 0.927 indicates that the model demonstrates a accurate result, with reasonable scatter around the diagonal and no trends in the residuals.

Table 3 Analysis of variance of the fitted second-order polynomial model for total extraction yields of isoflavones

\begin{tabular}{llllll}
\hline & $\begin{array}{l}\text { Sum of } \\
\text { Source }\end{array}$ & $\begin{array}{l}\text { Mean } \\
\mathrm{df}\end{array}$ & $F$ square & $p$-Value & Prob $>F$ \\
\hline Model & 0.3 & 9 & 0.033 & 5.35 & 0.0189 \\
$x_{1}$ & $4.50 \times 10^{-6}$ & 1 & $4.5 \times 10^{-6}$ & $7.28 \times 10^{-4}$ & 0.9792 \\
$x_{2}$ & 0.07 & 1 & 0.07 & 11.34 & 0.012 \\
$x_{3}$ & $5.18 \times 10^{-3}$ & 1 & $5.18 \times 10^{-3}$ & 0.84 & 0.3905 \\
$x_{1} x_{2}$ & $1.81 \times 10^{-3}$ & 1 & $1.81 \times 10^{-3}$ & 0.29 & 0.6056 \\
$x_{1} x_{3}$ & $5.57 \times 10^{-5}$ & 1 & $5.57 \times 10^{-5}$ & $9.01 \times 10^{-3}$ & 0.9271 \\
$x_{2} x_{3}$ & 0.017 & 1 & 0.017 & 2.82 & 0.1371 \\
$x_{1}{ }^{2}$ & 0.1 & 1 & 0.1 & 16.89 & 0.0045 \\
$x_{2}{ }^{2}$ & 0.012 & 1 & 0.012 & 2.01 & 0.1994 \\
$x_{3}{ }^{2}$ & 0.055 & 1 & 0.055 & 8.82 & 0.0208 \\
Residual & 0.043 & 7 & $6.18 \times 10^{-3}$ & - & - \\
Lack of fit & 0.022 & 3 & $7.28 \times 10^{-3}$ & 1.36 & 0.3752 \\
Pure error & 0.021 & 4 & $5.36 \times 10^{-3}$ & - & - \\
Cor total & 0.34 & 16 & - & - & -
\end{tabular}


Table 4 The predicted regression model in terms of coded factors from Box-Behnken program analysis

\begin{tabular}{|c|c|c|c|c|c|c|}
\hline Factor & $\begin{array}{l}\text { Coefficient } \\
\text { estimate }\end{array}$ & df & $\begin{array}{l}\text { Standard } \\
\text { error }\end{array}$ & $95 \%$ CI low & 95\% CI high & VIF \\
\hline Intercept & 10.16 & 1 & 0.043 & 10.06 & 10.27 & \\
\hline$x_{1}$ & 0.0075 & 1 & 0.031 & -0.06 & 0.087 & 1.06 \\
\hline$x_{2}$ & 0.096 & 1 & 0.032 & $6.98 \times 10^{-3}$ & 0.16 & 1.05 \\
\hline$x_{3}$ & 0.026 & 1 & 0.032 & -0.053 & 0.097 & 1.05 \\
\hline$x_{1} x_{2}$ & -0.021 & 1 & 0.043 & -0.14 & 0.057 & 1.05 \\
\hline$x_{1} x_{3}$ & 0.003632 & 1 & 0.043 & -0.094 & 0.11 & 1.05 \\
\hline$x_{2} x_{3}$ & -0.066 & 1 & 0.044 & -0.17 & 0.038 & 1 \\
\hline$x_{1}^{2}$ & -0.18 & 1 & 0.049 & -0.3 & -0.064 & 1.06 \\
\hline$x_{2}{ }^{2}$ & -0.054 & 1 & 0.043 & -0.14 & 0.062 & 1.01 \\
\hline$x_{3}{ }^{2}$ & -0.11 & 1 & 0.043 & -0.23 & -0.028 & 1.01 \\
\hline
\end{tabular}

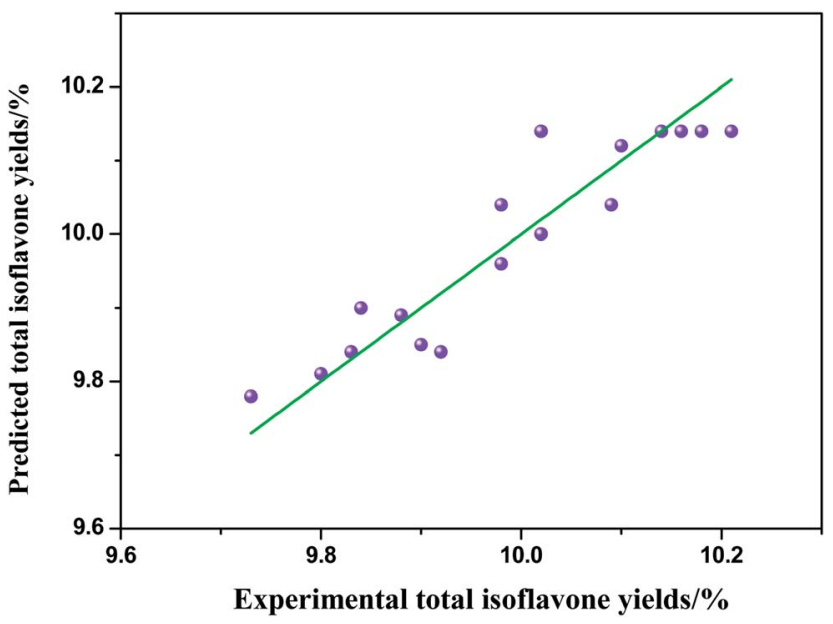

Fig. 5 Comparison of experimental and predicted total isoflavones yields from Puerariae lobata.

To consider the interactions between independent variables, 3D response surface plots are depicted in Fig. 6, showing how pairs of extraction parameters affect the extraction yields of total isoflavones from Puerariae lobata. All of the three surfaces are saddle-shaped, with a maximum point in the center of the experimental domain. Based on the 3D response surface plots, the optimum conditions for Puerariae lobata extraction were calculated to be as follows: extraction temperature of $120{ }^{\circ} \mathrm{C}$, extraction time of $45 \mathrm{~min}$ and solid/liquid ratio of $1: 15$. Under these optimum extraction conditions, the total isoflavone yields of three replicates were $10.21 \pm 0.34 \%, 9.91 \pm 0.41 \%$ and 10.03 $\pm 0.51 \%$, and the average was $10.05 \pm 0.42 \%$, which is very close to the predicted value of $10.04 \pm 0.41 \%$; this demonstrates that the model showed a good fit with the experimental values.

\subsection{Comparison of different extraction methods on the total isoflavone yields from Puerariae lobata}

To investigate the extraction effect of subcritical water, the extraction results obtained using this process were compared with those obtained by $70 \%$ ethanol reflux and ultrasonic extraction. Table 5 shows the extraction conditions and total
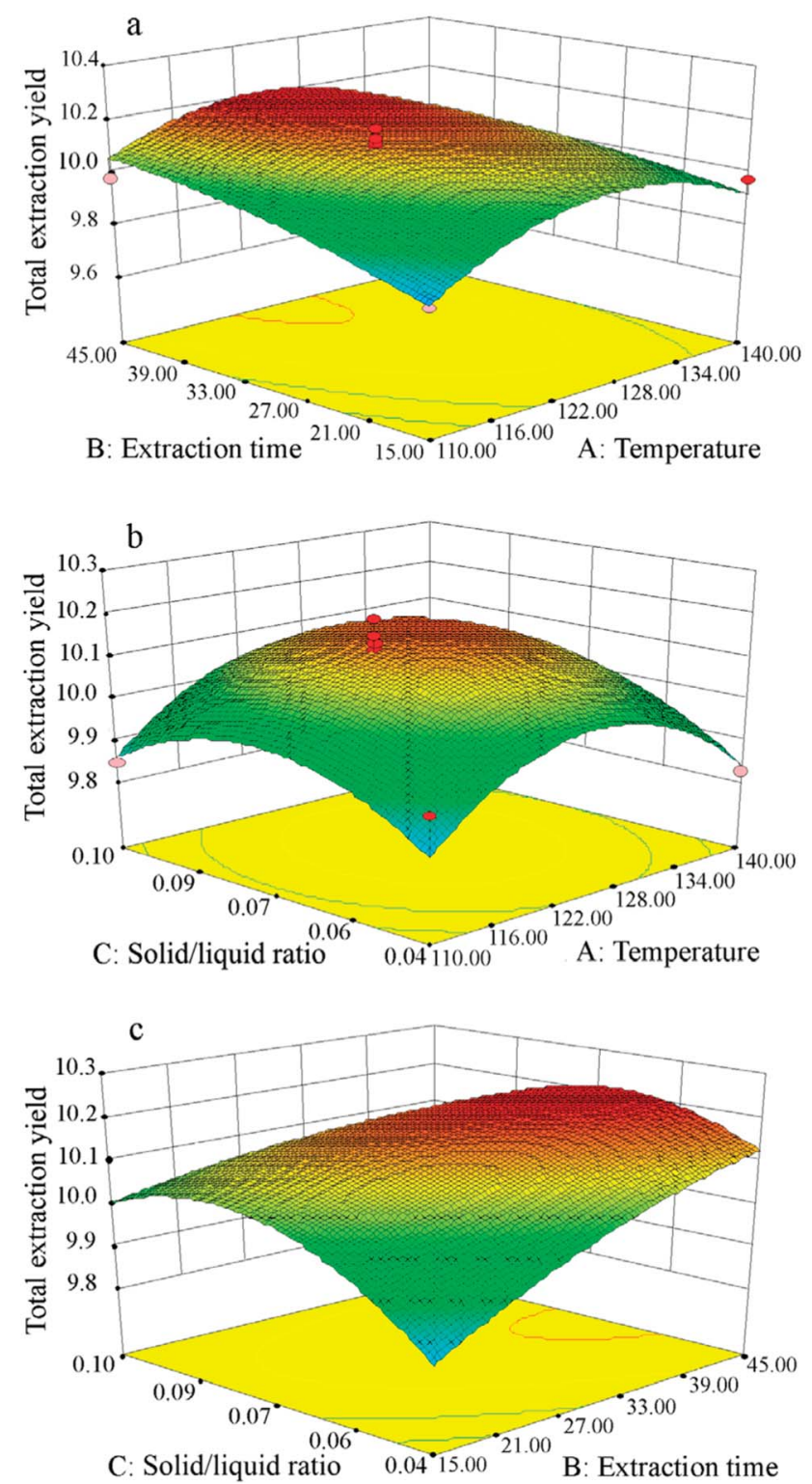

Fig. 6 Response surface plots showing interactions between experimental parameters on the total isoflavone yields from Puerariae lobata: (a) extraction time ( $\mathrm{min}$ ) and temperature $\left({ }^{\circ} \mathrm{C}\right)$; (b) solid/liquid ratio and temperature $\left({ }^{\circ} \mathrm{C}\right)$; (c) solid/liquid ratio and extraction time (min).

Table 5 Comparison of the total isoflavone yields obtained from Puerariae lobata with different extraction methods

\begin{tabular}{lllll}
\hline & \multicolumn{2}{l}{} & \multicolumn{2}{l}{$\begin{array}{l}\text { Extraction } \\
\text { Extraction method }\end{array}$} \\
\hline Reflux extraction & $90{ }^{\circ} \mathrm{C}$ & $120 \mathrm{~min}$ & $1: 40$ & $9.21 \pm 0.36 \%$ \\
Ultrasonic extraction & $50{ }^{\circ} \mathrm{C}$ & $45 \mathrm{~min}$ & $1: 30$ & $9.64 \pm 0.23 \%$ \\
SCWE & $120^{\circ} \mathrm{C}$ & $35 \mathrm{~min}$ & $1: 15$ & $10.05 \pm 0.42 \%$
\end{tabular}

isoflavone extraction yields. It can be seen that the extraction yields with SCWE were the highest, but reflux and ultrasonic extraction had extraction yields that were almost as high. The extraction time for SCWE was shorter than those for reflux and 
ultrasonic extraction. Besides, the consumption of extraction solvent by SCWE was less than those for the conventional methods. This is probably due to the more complete diffusion of water molecules into the particles of the herbal materials. Therefore, it is clear that subcritical water is an effective extraction solvent and that SCWE could utilize less solvent and take a shorter time than other extraction methods. In addition, as shown in Table 5, no significant differences in the isoflavone yields produced by the three different methods were found.

\section{Conclusion}

In this study, the extraction from Puerariae lobata of four individual isoflavones, namely puerarin, $3^{\prime}$-methoxypuerarin, daidzin and daidzein, as well as that of the total isoflavones were studied using subcritical water. The extraction temperature, extraction time and solid/liquid ratio were optimized by singlefactor experiments for the four isoflavones and the total isoflavone yield was optimized by RSM. Compared with conventional extraction methods, SCWE provides a higher extraction efficiency due to its high diffusion velocity. The presented results demonstrate that subcritical water is an excellent extractant and that SCWE is a simple, fast, environmentally friendly extraction method which has a low consumption of solvent.

\section{Conflicts of interest}

The authors declare that there is no conflict of interest regarding the publication of this paper.

\section{Acknowledgements}

The authors acknowledge financial support from the National Natural Science Foundation of China (No. 21703126) and the Shandong Keypoint Research \& Development Plan (2018GSF117006).

\section{References}

1 Y. G. Chen, Y. L. Song, Y. Wang, Y. F. Yuan, X. J. Huang, W. C. Ye, Y. T. Wang and Q. W. Zhang, J. Pharm. Biomed. Anal., 2014, 93, 51-58.

$2 \mathrm{~W}$. Cherdshewasart and W. Sutjit, Phytomedicine, 2008, 15, 38-43.

3 M. E. Xu, S. Z. Xiao, Y. H. Sun, X. X. Zheng, Y. Ou-Yang and C. Guanm, Life Sci., 2005, 77, 3183-3196.

4 S. M. Boue, T. E. Wiese, S. Nehls, M. E. Burow, S. Elliott, C. H. Carter-Wientjes, B. Y. Shih, J. A. Mclachlan and T. E. Cleveland, J. Agric. Food Chem., 2003, 51, 2193-2199.

5 F. L. Hsu, I. M. Liu, D. H. Kuo, W. C. Chen, H. C. Su and J. T. Cheng, J. Nat. Prod., 2003, 66, 788-792.
6 R. W. Jiang, K. M. Lau, H. M. Lam, W. S. Yam, L. K. Leung, K. L. Choi, M. M. Y. Waye, T. C. W. Mak, K. S. Woo and K. P. Fung, J. Ethnopharmacol., 2005, 96, 133-138.

7 S. G. Ru, D. Chen and W. Wang, Period. Ocean Univ. China, 2015, 45, 31-36.

8 J. S. Eun, S. Y. Kyung and M. Byung-Sun, Arch. Pharmacal Res., 2012, 35, 823-837.

9 S. E. Jin, Y. K. Son, B. Min, H. A. Jung and J. S. Choi, Arch. Pharmacal Res., 2012, 35, 823-837.

10 Y. Wang, L. Yin, Y. Li, P. Liu and Q. Cui, Clin. Orthop. Relat. Res., 2008, 466, 1059-1067.

11 L. Mei-Hwa and L. Chuan-Chuan, Food Chem., 2007, 105, 223-228.

12 T. Yasuda, M. Endo, T. Kon-no, T. Kato, M. Mitsuzuka and K. Ohsawa, Biol. Pharm. Bull., 2005, 28, 1224-1228.

13 W. L. Dong, L. Changho, K. In-Ho and T. K. Yun, Molecules, 2013, 18, 10404-10412.

14 K. Min-Jung, C. Chan-Ick, C. Sang-Woo and C. Myong-Soo, J. Food Eng., 2011, 102, 327-333.

15 J. T. He, Z. W. Zhao, Z. H. Shi, M. P. Zhao, Y. Z. Li and W. B. Chang, J. Agric. Food Chem., 2005, 53, 518-523.

16 S. Gbashi, O. A. Adebo, L. Piater, N. E. Madala and P. B. Njobeh, Sep. Purif. Rev., 2017, 46, 21-34.

17 D. Bianca, J. Jeremy, C. Jamuna, K. B. Liam, N. S. James, K. Nathan, S. Miguel, G. Nuri, B. Alex and S. Jason, ChemistrySelect, 2017, 2, 2439-2443.

18 W. J. Kim, B. Veriansyah, Y. W. Lee, J. Kim and J. D. Kim, J. Ind. Eng. Chem., 2010, 16, 425-430.

19 S. D. Allmon and J. G. Dorsey, J. Chromatogr. A, 2010, 1217, 5769-5775.

20 F. Liu, E. S. Ong and S. F. Y. Li, Food Chem., 2013, 141, 18071813.

21 E. Ibaňez, A. Kubátová, F. J. Seňoráns, S. Cavero, G. Reglero and S. B. Hawthorne, J. Agric. Food Chem., 2003, 51, 375-382.

22 Z. Zekovic, S. Vidovic, J. Vladic, R. Radosavljevic, A. Cvejin and P. Mohamed, J. Supercrit. Fluids, 2014, 95, 560-566.

23 G. Derringer, Journal of Quality Technology, 1980, 12, 214219.

24 D. Bas, J. Food Eng., 2007, 78, 836-845.

25 C. Y. Gan and A. A. Latiff, Food Chem., 2011, 124, 1277-1283.

26 M. A. Bezerra, R. E. Santelli, E. P. Oliveira, L. S. Villar and L. A. Escaleira, Talanta, 2008, 76, 965-977.

27 M. Hisashi, M. Toshio, X. Fengming, N. Kiyofumi, Y. Masayuki, B. Yan, D. M. Xing, Y. Ding, J. L. Tao and L. J. Du, J. Pharm. Biomed. Anal., 2005, 37, 297-301.

28 D. Y. Zhu, China Brewing, 2015, 11, 145-148.

$29 \mathrm{H}$. T. Li, Study on extraction technology of effective components of Puerariae and its antialcoholic effect, Northeast Agricultural University, Harbin, 2006. 